\title{
International quality control of phage typing of Staphylococcus aureus
}

\author{
R. R. MARPLES (Secretary), V. T. ROSDAHL* (Chairman) and Members of the IUMS \\ Subcommittee† \\ Laboratory of Hospital Infection, PHLS Central Public Health Laboratory, 61 Colindale Avenue, London NW9 \\ $5 H T$ and ${ }^{*}$ StatensSerum Institute, 5 Artillerivej, 2300 Copenhagen S, Denmark
}

\begin{abstract}
A questionnaire was sent to the 48 national typing centres for Staphylococcus aureus and 31 replies were received. Methods of phage typing varied and molecular methods were not universally available, although pulsed-field gel electrophoresis was offered by 13 centres. Results for a quality control phage typing exercise were received from 25 centres. Increased standardisation of methods and definitions are indicated. Differences from the consensus patterns were mainly due to typing at an inappropriate dilution of phage, but five strains caused difficulties in many centres. Overall reproducibility was good. Phage typing remains a cost-effective method for epidemiological studies, particularly on a large scale. The strains selected for the quality control exercise included many strains suitable for controlling molecular methods as well as testing phage typing. Molecular methods help in the validation of the conclusions which may be drawn from phage typing.
\end{abstract}

\section{Introduction}

Bacteriophage typing of Staphylococcus aureus has been organised internationally since 1953 and regular

Received 17 Sept. 1996; accepted 2 Nov. 1996.

Corresponding author: Dr R. R. Marples.

$\dagger$ †.A.N. Pessat, Universidad Nacional de Cordoba, Argentina; A. Vickery, Royal Prince Alfred Hospital, Australia; C. Godard, Institut Pasteur, Belgium; E. M. Mamizuka, Universidade de Sao Paulo, Brazil; K. Toshkova, National Centre of Infectious and Parasitic Diseases, Bulgaria; W. M. Johnson, Laboratory Centre for Disease Control, Canada; Zhu Chao, Chengdu Institute, China; P. Petras, National Institute of Public Health, Czech Republic; B. D. Cookson, CPHL, London, UK; J. Vuopio-Varkila, National Public Health Institute, Finland; N. El Solh, Institut Pasteur, France; K. P. Schaal, Rheinische Friedrich-Wilhelm-Universitat, Germany; W. P. Mak, Sai Ying Pun Polyclinic, Hong Kong; H. Milch, National Institute of Hygiene, Hungary; M. D. Mathur, Maulana Azad Medical College, India; Z. Samra, Tel Aviv University, Israel; S. Iyobe, Gunma University, Japan; D. T. Cho, Kyungpook University, Korea; E. E. Udo, Kuwait University, Kuwait; Y. A. Hanifah, Universiti Malaya, Malaysia; W. J. van Leeuwen, RIVM, The Netherlands; H. Heffernan, ESR Health, New Zealand; J. Galinski, Medical Academy of Gdansk, Poland; J. A. G. Melo Cristino, Lisbon, Portugal; M. Negut, Institutul Cantacuzino, Romania; O. Dmitrenko, Gamaleya Institute, Russia; A. O. Osoba, Riyadh Armed Forces Hospital, Saudi Arabia; K. P. Klugman Institute for Medical Research, South Africa; A. Vindel, Instituto de Salud Carlos III, Spain; R. Sunthadvanich, Nonthaburi, Thailand. quality control exercises have been performed by the WHO Reference Laboratory for the IUMS Subcommittee for Phage Typing of Staphylococci. Detailed results have not been published previously as results were compared with those of the reference laboratory. The Subcommittee decided that, in view of the increasing emphasis on quality assurance, accreditation and the use of alternative molecular typing methods particularly for methicillin-resistant $S$. aureus (MRSA) - the national typing centres should report on their facilities for such methods and that the standard for the quality control exercise should be the consensus result.

Since the general acceptance of methods of phage typing of $S$. aureus [1-3] the importance of quality control has always been stressed. The phages in the International Set have been reviewed regularly, with the addition of phages 94, 95 and 96 in 1974 [4]. The recommended layout of a phage typing test is 25 phage application sites, in five rows of five, on a nutrient agar plate flooded with the isolate to be typed. The number of phages in the International Set has been restricted to 23 , to leave room for extra phages found to be useful locally. Currently, international studies of supplementary phages for typing methicillin-resistant isolates are in progress. The International Set phages and appropriate propagating strains are replaced from the Reference Laboratory at 4-year intervals.

At the meeting of the Subcommittee in Stockholm in 
1992, it was decided to institute a questionnaire survey of typing methods and a quality control exercise involving the circulation of selected strains for assessing, firstly, phage typing and, later, other typing methods [5]. These strains were lyophilised and circulated in 1994 to participating national centres. This paper describes the results of both the questionnaire and the quality control exercise.

\section{Materials and methods}

\section{Questionnaire}

This addressed several distinct areas and was sent to the 48 centres on the mailing list. The first set of questions related to phage typing, including the annual workload, the number of centres and hospitals served and the criteria used for typing. The routine method of typing was queried: were isolates typed first at routine test dilution (RTD: that dilution of phage that gave just confluent lysis on the designated propagating strain) and retested with phage preparations 100 times stronger than RTD $($ RTD $\times 100)$ ? Details of the typing methods used, local phages and a breakdown of the typing results were requested, along with the level of computerisation. A second series of questions explored the availability of additional phage-based and molecular typing methods. A final set of questions addressed other phenotypic characters, including antibiotic susceptibility tests, frequency of MRSA, toxin detection tests and whether coagulase-negative staphylococci were typed.

\section{Selection of strains for the quality control exercise}

Twenty-one strains were selected (Table 1), including nine from a comparative pulsed-field gel electrophoresis (PFGE) study of MRSA [6], two from an outbreak of staphylococcal scalded skin syndrome [7] and several control strains. Table 1 also lists the identifying numbers given to the strains common to the study reported in reference 6 . Two strains were included twice to permit a measure of reproducibility. The strains were lyophilised in Denmark and distributed by the CPHL in London to the 36 centres that indicated a wish to participate.

\section{Methods of analysis}

The results with the phages of the International Set were entered into a computer and analysed by inspection of the printouts. For the quality control results the forms were interpreted by RRM and coded numerically as follows: $1,( \pm)$ indicating $<10$ plaques; 2 , ( \pm ) indicating $10<20$ plaques; $3,(+)$ indicating 20-50 plaques; 7 , intermediate result; $8,(++)$ indicating $>50$ plaques; 9 , confluent lysis (CL) or opaque lysis (OL); 0 , inhibition reaction.

The product of staphylococcal phage typing is a pattern of reactions of varying strengths rather than a reaction with a single phage as in some other systems. It is a fully tested convention that two strains are considered 'different' if two or more phages give a++ reaction on one strain and no reaction on the other strain at RTD [8]. Weak reactions are important in preventing the acceptance of a strong reaction difference (srd) in this system, but are not reported in the abbreviated phage type except as a terminal ' + '. The recommended method to overcome this difficulty is to photocopy the worksheet and to comment on the interpretation [2].

The codes were printed out by strain at RTD, at RTD $\times 100$ and a consensus 'correct' pattern at the appropriate dilution was assigned. The centres showing no strong reaction difference (srd), one srd and

Table 1. Strains selected for the quality control exercise

\begin{tabular}{|c|c|c|c|c|c|}
\hline Strain no. & Origin & Number & $\begin{array}{c}\text { Methicillin } \\
\text { susceptibility* }\end{array}$ & Comment & ID no.* \\
\hline $1+7$ & France & BM9290 & $\mathrm{R}$ & French epidemic MRSA & \\
\hline 2 & Holland & BAC 170190 & $\mathrm{~S}$ & Control strain for group I and III phages & \\
\hline 3 & England & $\mathrm{E} 12 \mathrm{M} 258$ & $\mathrm{R}$ & EMRSA-12 & 4 \\
\hline 4 & England & $85 / 1836$ & $\mathrm{R}$ & German MRSA & 8 \\
\hline 5 & Germany & $530 / 92$ & $\mathrm{~S}$ & Group I control & \\
\hline 6 & England & E03/M307 & $\mathrm{R}$ & EMRSA-3 & 3 \\
\hline 8 & England & ST90/7340 & $S$ & ETB producer & \\
\hline 9 & England & $84 / 9580$ & $\mathrm{R}$ & South African MRSA & 6 \\
\hline 10 & England & $85 / 5484$ & $\mathrm{R}$ & Saudi Arabian MRSA & 12 \\
\hline 11 & Denmark & 10 & $\mathrm{~S}$ & Singapore MSSA & \\
\hline $12+18$ & England & $\mathrm{NC} 10442$ & $\mathrm{R}$ & Original MRSA & 1 \\
\hline 13 & Denmark & 19627 & $\mathrm{~S}$ & Type 95 control & \\
\hline 14 & Denmark & W57 & $\mathrm{S}$ & Control strain & \\
\hline 15 & England & $\mathrm{NC} 12232$ & $\mathrm{R}$ & EMRSA-2 & 2 \\
\hline 16 & England & ST $90 / 8556$ & $\mathrm{~S}$ & ETA producer & \\
\hline 17 & Denmark & 19609 & $\mathrm{~S}$ & Group V control & \\
\hline 19 & England & $85 / 4670$ & $\mathrm{R}$ & Canadian MRSA & 11 \\
\hline 20 & Germany & $616 / 92$ & $\mathrm{~S}$ & Type 29 control & \\
\hline 21 & England & $84 / 5528 / 1$ & $\mathrm{R}$ & EMRSA-1 & 5 \\
\hline
\end{tabular}

$\mathrm{R}$, resistant; $\mathrm{S}$, sensitive.

${ }^{*}$ Identification number as used in reference 6 . 
two or more srd from the 'correct' pattern were tabulated. The 'errors' were reviewed and the importance of each was assessed.

\section{Results}

\section{Questionnaire}

Number and activity of national centres. Thirty-one centres replied to the questionnaire. Four indicated that phage typing had been discontinued and there had been centralisation of phage propagation for Scandinavia in Copenhagen. Twenty-seven centres returning the questionnaire claimed to be active and three other centres were known to be typing. Most centres offered a national typing service but this was also delegated to multiple local centres in England and Wales, Hungary, Russia, Sweden and Thailand. Seventeen centres served $>10$ hospitals (median 20), but five served less than this number.

Centres were asked to record the number of isolates typed in 1993. While two centres typed $>10000$ isolates a year and 11 centres typed between 1000 and 9999, 13 centres typed $<1000$ isolates in 1993.

Methods of phage typing. The recommended methods for phage typing have not changed since 1972 [2]. The pure culture should be typed at RTD and, if no strong reaction ( $>50$ plaques) is obtained, retested the next day at RTD $\times 100$. The medium is not standardised and although a simple nutrient agar is accepted as satisfactory, the addition of $\mathrm{CaCl}_{2} 400 \mathrm{mg} / \mathrm{L}$, is recommended [2].

Only seven centres typed at RTD initially and retyped at $\mathrm{RTD} \times 100$ when $\mathrm{a}++$ reaction was not present at RTD, while 17 routinely typed initially at both dilutions. Four centres typed only at RTD $\times 100$ and not at RTD at all, and one centre retyped at RTD $\times 125$. The medium used was very variable, but eight centres mentioned supplementing it with $\mathrm{CaCl}_{2}$. Criteria for submission of isolates for typing were not stringent and most centres typed on demand. Fifteen centres typed with phages additional to the International Set, mainly for MRSA.

Overall typability varied from country to country, ranging from 35 to $92 \%$ with a median of $78 \%$. Criteria for classifying an isolate as non-typable (NT) were not uniform; for instance, one centre called an isolate NT for failing to give a ++ result at RTD whereas another would only call an isolate NT if it gave no reaction after heat-shock. Classically, isolates were considered NT if no ++ reaction was found at RTD $\times 100$, but only two centres applied this definition.

MRSA. MRSA were reported by 24 centres and exceeded 1000 isolates, in 1993, in England, Israel and Spain, while more than 400 isolates were recorded by Belgium, Bulgaria, Germany, Malaysia, the Netherlands and Romania. Most MRSA isolates typed with the group III phages, but NT and I + III patterns were prominent.

Additional typing methods. All centres used antibiotic susceptibility results as additional markers, testing a mean of 15.7 , with a range of $8-27$, antibiotics. Reverse typing (assessing the host range of carried phage) was a standard method for nine centres and another nine centres offered heat shock typing. Biotyping of MRSA had been used by seven centres but details were not given.

Protein typing. This approach to typing was much less favoured than DNA studies, with only three centres routinely studying proteins. One centre examined whole cell proteins, another extracellular proteins and two offered Western blotting.

DNA studies. Nineteen centres could carry out plasmid analyses and 11 also performed restriction enzyme analysis of the plasmid DNA. Restriction fragment length polymorphism on whole cell DNA was available in seven centres and pulsed-field gel electrophoresis in a contour-clamped homogeneous electric field (CHEF) was available in 13 centres.

Ribotyping was used in eight centres, insertion sequences by three and probing for the mecA gene and other PCR methods by nine. Nine centres reported no capability to carry out DNA studies, four studied plasmids only and of the 18 with greater capabilities, reliance on CHEF alone (three centres) or on PCR alone (two centres) was reported.

Toxins. Staphylococcal enterotoxin A (SEA), SEB, SEC and SED detection was offered by 10 centres and six also tested for SEE production. Toxic shock syndrome toxin-1 (TSST-1) detection was offered in 11 centres and the exfoliative toxins, ETA and ETB, by seven. Only five centres offered tests for all three groups of toxins.

\section{Quality control exercise}

Twenty-five centres returned typing results for the 21 test isolates, including two centres that did not return the questionnaire. The centres had been asked to type the strains at RTD and at RTD $\times 100$, but four centres did not type at RTD at all and several did not type at RTD $\times 100$ if a ++ reaction was recorded at RTD, the correct method for routine typing, but not for the exercise. Only 12 typed all the strains at both RTD and RTD $\times 100$. Most returned copies of the worksheets but some reported the summary phage patterns without minor reactions. These differences precluded a comprehensive mathematical analysis of the results. 
Errors by isolate. The overall results are summarised in Table 2 which lists the strain number, the optimum titre of phage, the modal pattern, the number of centres with no, one or more than one strong reaction difference (srd) from the modal pattern and the percentage of centres for each strain that would not have distinguished their results from the modal pattern using the two srd rule.

For 16 of the 21 isolates, the results showed $80 \%$ or better, by centre, agreement with the 'correct' pattern, i.e., were indistinguishable from the modal pattern by the two srd rule; for 11 isolates, results were $90 \%$ or better. Strains $6,8,10,11$ and 15 appeared to cause problems in several laboratories, suggesting that the difficulties lay in the strain rather than the laboratory. Four centres recorded additional opaque lysis reactions with strains 4,10 and 21 .

Strains. Comments on each strain include reasons for the choice of the 'correct' pattern and common variations. These strains are intended to be available as control strains, but care must be taken as several have caused major outbreaks and are not benign (Table 1).

Strains $1+7$ gave a clean pattern of 77 at RTD with few outlying reactions. At RTD $\times 100,11$ of the 20 centres testing at this strength recorded a reaction with phase 84 .

Strain 2 gave a long I + III pattern at RTD. At RTD $\times 100$, group II phages became prominent. The consensus pattern was: $29 / 52 / 52 \mathrm{~A} / 80 / 95 / 6 / 42 \mathrm{E} / 47 / 53$ / 54/75/77/83A/84/85/81/96.

Strain 3 gave a clean pattern of $75 / 83 \mathrm{~A}$ at RTD, but at RTD $\times 100$ reactions with phages 54 and 77 were found by the majority of centres.

Strain 4: the pattern NT at RTD $\times 100$ was recorded by 11 of 23 and was taken as the 'correct' pattern over 84 , recorded by nine centres.

Strain 5: the pattern, $52 / 80$ at RTD, needed the proviso that a weak reaction with phage $52 \mathrm{~A}$ was seen in 13 of the 21 centres testing. This reaction became strong at RTD $\times 100$.

Strain 6: a reasonably clean pattern of $75 / 83 \mathrm{~A}$ at RTD $\times 100$ was seen, but five centres found it NT and five detected a reaction, strong in three, with phage 84 .

Strain 8: the pattern $3 \mathrm{~A} / 71$ at RTD was chosen as 'correct'. Only 71 was reported as a strong reaction by 14 of 21 centres while phage $3 \mathrm{~A}$ gave a strong reaction in only five of the 11 centres with any reaction with this phage. All but one strong reaction were within group II.

Strain 9: 17 of 25 centres found this strain NT at RTD $\times 100$, but four recorded a ++ reaction with phage 75 and three others a weak reaction with this phage.

Strain 10: NT at RTD $\times 100$ was chosen over 85 , as

Table 2. Modal patterns and typing results by strain

\begin{tabular}{|c|c|c|c|c|c|c|}
\hline \multirow{2}{*}{$\begin{array}{l}\text { Strain } \\
\text { no. }\end{array}$} & \multirow{2}{*}{$\begin{array}{l}\text { Optimal } \\
\text { phage } \\
\text { dilution }\end{array}$} & \multirow{2}{*}{$\begin{array}{l}\text { Modal } \\
\text { 'correct' } \\
\text { pattern }\end{array}$} & \multicolumn{3}{|c|}{$\begin{array}{l}\text { Number of centres with the stated } \\
\text { srds different from 'correct' }\end{array}$} & \multirow{2}{*}{$\begin{array}{l}\text { Percentage } \\
\text { 'correct"* }\end{array}$} \\
\hline & & & 0 & 1 & $>1$ & \\
\hline 1 & RTD & 77 & 13 & 5 & 3 & 86 \\
\hline 2 & RTD & $\mathrm{I}+\mathrm{III}^{\dagger}$ & 10 & 9 & 2 & 90 \\
\hline 3 & RTD & $75 / 83 \mathrm{~A}$ & 13 & 7 & 1 & 95 \\
\hline 4 & $\mathrm{RTD} \times 100$ & NT & 11 & 8 & 4 & 83 \\
\hline 5 & RTD & $52 / 80+$ & 16 & 3 & 2 & 90 \\
\hline 6 & $\mathrm{RTD} \times 100$ & $75 / 83 \mathrm{~A}$ & 7 & 10 & 7 & 71 \\
\hline 7 & RTD & 77 & 14 & 6 & 1 & 95 \\
\hline 8 & RTD & $3 \mathrm{~A} / 71$ & 5 & 10 & 6 & 71 \\
\hline 9 & $\mathrm{RTD} \times 100$ & NT & 21 & 4 & 0 & 100 \\
\hline 10 & $\mathrm{RTD} \times 100$ & NT & 12 & 5 & 7 & 71 \\
\hline 11 & RTD & 53 & 7 & 7 & 7 & 67 \\
\hline 12 & RTD & III $^{\dagger}$ & 15 & 5 & 1 & 95 \\
\hline 13 & RTD & 95 & 20 & 1 & 0 & 100 \\
\hline 14 & RTD & $\mathrm{I}+\mathrm{III}^{\dagger}$ & 8 & 9 & 4 & 81 \\
\hline 15 & $\mathrm{RTD} \times 100$ & $80 / 85$ & 5 & 6 & 12 & 48 \\
\hline 16 & $\mathrm{RTD} \times 100$ & $3 \mathrm{~A} / 3 \mathrm{C}$ & 14 & 6 & 5 & 80 \\
\hline 17 & RTD & $94 / 96$ & 18 & 2 & 1 & 95 \\
\hline 18 & RTD & III $^{\dagger}$ & 14 & 5 & 2 & 90 \\
\hline 19 & $\mathrm{RTD} \times 100$ & 84 & 11 & 11 & 2 & 92 \\
\hline 20 & RTD & 29 & 16 & 3 & 1 & 95 \\
\hline 21 & RTD $\times 100$ & 85 & 10 & 10 & 5 & 80 \\
\hline
\end{tabular}

RTD, routine test dilution; srd, strong reaction difference.

*Results within 1 srd of the modal pattern were included as 'correct'.

${ }^{\dagger}$ See Results section of text. 
only seven of the 12 reactions with this phage were strong, including two centres with multiple CL reactions that were clearly in error.

Strain 11: the simple pattern of 53 at RTD was chosen over $53 / 83 \mathrm{~A} / 85$ at $\mathrm{RTD} \times 100$ as this minimised the number of centres recording more than two srd.

Strains $12+18$ : the strong group III pattern at RTD was well achieved. The full pattern was: $47 / 53 / 54 / 75 /$ $77 / 84 / 85$.

Strain 13: this typical 95 strain gave few problems.

Strain 14: this strain typed with a long group I + III pattern at RTD. Differences seen were mainly in the group I reactions. The consensus pattern was: $29 / 52 /$ 52A/95/6/42E/54/75/77/84/85/81.

Strain 15: the minimum pattern of $80 / 85$ at RTD $\times 100$ was chosen as 'correct', but the strain is clearly susceptible to other group I phages. Including $52,52 \mathrm{~A}$ and 79 in the pattern worsened the result even more than the $48 \%$ with one srd or less with the accepted pattern.

Strain 16: a weak group II pattern of $3 \mathrm{~A} / 3 \mathrm{C}$ at RTD $\times 100$ was chosen as 'correct'. A reaction with phage 55 was recorded by 11 of the 25 centres, but in only four was this reaction strong. The distinction of this strain from strain 8 by the exfoliative toxin produced was critical in the understanding of the original study [7].

Strain 17: there were few problems with this group V strain which typed well as 94/96 at RTD.

Strain 19: the chosen pattern of 84 at RTD $\times 100$ allowed a NT result to count as one srd.

Strain 20: the pattern of 29 at RTD was chosen, although four centres found it NT at this dilution.

Strain 21: a pattern of 85 at RTD $\times 100$ was chosen with nine of 15 centres with any reaction with this phage scoring it as strong. This strain has been associated with strain 10 [6].

Reproducibility. When the results of each centre for strain 1 and 7 were examined, two centres recorded two srd between the repetitions at RTD, the recommended dilution, and two further centres showed such a discrepancy at RTD $\times 100$. The RTD differences could have been recording errors as the results were 75 and 77 for one centre and 77 and $83 \mathrm{~A}$ for the other. These pairs of phages are normally contiguous. At RTD $\times 100$, one centre found one of the pair of strains NT, raising the possibility that the strain did not grow or was contaminated; no explanation was obvious for the other centre's findings. No such discrepancy was seen in the repetition of strains 12 and 18. This gives an overall reproducibility of $91.3 \%$.

Errors by centre. Most errors recorded arose from typing with an inappropriate dilution of phage. Four centres typed only at RTD $\times 100$ while RTD was the recommended strength in 13 of the 21 strains. Inevitably, several srd had to be scored for these centres, although the results were often close to the consensus at RTD $\times 100$. Similarly, with centres with titres of phage apparently on the low side, recording NT at RTD and retyping at RTD $\times 100$ led to problems in interpretation. Other centres accepted $\mathrm{a}++$ reaction with an additional phage not in the International Set as a type at RTD and did not retype the strain at RTD $\times 100$. Seven centres showed no significant errors. Five appeared to have lower titre phages and two higher than the consensus. The number of individual complete errors was small. Often contiguous phages were affected and this may have been caused by recording error or misfilling the typing block.

\section{Discussion}

Phage typing of $S$. aureus became organised internationally in 1953 and was widely accepted, in part because of the worldwide spread of a strain typing as $80 / 81$ [2]. The subcommittee controlling phage typing internationally decided that all laboratories would type all isolates with the agreed International Set, now including 23 phages, although the use of additional and experimental phages was not precluded. The methods and interpretation of the results were standardised [1] and comparisons of the results on test strains with those of the reference laboratory were regularly performed. In recent years molecular methods have been advocated and compared favourably with phage typing $[9,10]$. A combination of two typing methods may be most efficacious [9]. The effort required to maintain the ability for phage typing has been emphasised, but phage typing itself is cheap [10] particularly when extended to large numbers of isolates. The number of isolates that can be studied by PFGE is limited, indeed the CDC criteria for PFGE typing [10] include one stipulating that all isolates must be sent in one shipment, as accurate interpretation requires (them) to be tested on the same gel. Where the same strain is prevalent in many hospitals such as EMRSA-15 and EMRSA-16 in England [11], phage typing backed up with PFGE was needed to handle the workload of thousands of isolates per month.

There is a need to attempt to upgrade the standardisation of the methods of phage typing as variation has developed over the years. A standard typing medium could now be investigated and the amount of calcium could be defined. Criteria for non-typability could be agreed and the possibility that some phages are more discriminatory than others should be pursued. Weak 
reactions, if reproducible, may be more significant than is now accepted, especially when substantiated by other methods such as PFGE. These questions should be considered by the Subcommittee.

The low numbers typed by many national centres suggests that the availability of phage typing as the quickest and cheapest method for the initial investigation of an outbreak due to $S$. aureus has not been adequately publicised. In fact, typing only occasionally makes it difficult to ensure satisfactory quality control. Phage typing has been the classical method for detecting epidemic and pandemic strains, although confirmation of selected isolates by molecular methods is now needed. The ability of the national centres to provide molecular follow-up studies was very varied, but nearly half the centres could produce acceptable molecular support for phage and other phenotypic characterisation. The interplay between the typing laboratory and the hospital infection control teams is essential for successful epidemiological control of an epidemic.

Variations in the methods of phage typing have evolved and a main message from this quality control exercise was that typing at both routine test dilution (RTD) and non-typable isolates at RTD $\times 100$ should remain the recommended procedure. Criteria for typing recorded in the questionnaire were lax; this may change with increased financial stringency. The importance of stating a clear epidemiological question must be stressed [10].

Overall the quality control exercise indicated good comparability between national centres, but some technical errors were seen. Standardisation of media, calcium content and criteria of typability need to be agreed. Centres are encouraged to re-assess their typing methods such that the consensus result is obtained on retyping the test strains. The test strains should become useful control strains, not only for phage typing, but also for molecular methods.

\section{References}

1. Blair JE, Williams REO. Phage typing of staphylococci Bull World Health Organ 1961; 24: 771-784.

2. Parker MT. Phage-typing of Staphylococcus aureus. In: Norris JR, Ribbons DW (eds) Methods in microbiology, vol $7 \mathrm{~B}$. London, Academic Press. 1972: 1-28.

3. Richardson JF, Noble WC, Marples RR. Species identification and epidemiological typing of the staphylococci. In: Board RG, Jones D, Skinner FA (eds) Identification methods in applied and environmental microbiology. Society for Applied Bacteriology Technical Series no. 29. Oxford, Blackwell Scientific Publications. 1992: 193-219.

4. Asheshov EH, Skalova R. International Committee on Systematic Bacteriology. Subcommittee on the phage-typing of staphylococci. Minutes of the meeting, 26 September 1974. Int J Syst Bacteriol 1975; 25: 233-234.

5. Marples RR. Extraordinary meeting of the International Union of Microbiological Societies Subcommittee on the phagetyping of Staphylococci. Minutes of the meeting, 30 June 1992. Int J Syst Bacteriol 1993; 43: 187.

6. Cookson BD, Aparicio P, Deplano A, Struelens M, Goering R, Marples R. Inter-centre comparison of pulsed-field gel electrophoresis for the typing of methicillin-resistant Staphylococcus aureus. J Med Microbiol 1996; 44: 179-184.

7. Dave J, Reith S, Nash JQ, Marples RR, Dulake C. A double outbreak of exfoliative toxin-producing strains of Staphylococcus aureus in a maternity unit. Epidemiol Infect 1994; 112: $103-114$.

8. Williams REO, Rippon JE. Bacteriophage typing of Staphylococcus aureus. J Hyg 1952; 50: 320-353.

9. Tenover FC, Arbeit R, Archer G et al. Comparison of traditional and molecular methods of typing isolates of Staphylococcus aureus. J Clin Microbiol 1994; 32: 407-415.

10. Bannerman TL, Hancock GA, Tenover FC, Miller JM. Pulsedfield gel electrophoresis as a replacement for bacteriophage typing of Staphylococcus aureus. J Clin Microbiol 1995; 33: $551-555$.

11. Anon. Epidemic methicillin resistant Staphylococcus aureus. Commun Dis Rep CDR Weekly 1996; 6: 197. 